\title{
Investigation of Water-in-Biodiesel Emulsion Characteristics Produced by Ultrasonic Homogenizer
}

\author{
Zainal Ambri Abdul Karim ${ }^{1 *}$, Degen Tee ${ }^{1}$, Mohammed Yahaya $\mathrm{Khan}^{2}$, Ftwi Yohaness \\ Hagos $^{3}$ \\ ${ }^{1}$ Department of Mechanical Engineering, Universiti Teknologi PETRONAS, 32610 Seri Iskandar, \\ Perak, Malaysia. \\ ${ }^{2}$ Department of Mechanical Engineering, Ilahia College of Engineering and Technology Mulavoor \\ (P.O), Muvattupuzha, Ernakulum District Kerala, Pincode-686673. India. \\ ${ }^{3}$ Faculty of Mechanical Engineering, Universiti Malaysia Pahang, 26600 Pekan, Pahang.
}

\begin{abstract}
Limited studies had been conducted using water-in-diesel emulsion produced from ultrasonic homogenizing method. In this study, Water-in-Biodiesel Emulsions (WiBE) produced using ultrasonic homogenizer were characterized and studied for their physical and chemical properties through various laboratory investigations. The data were then compared with WiBE produced using mechanical homogeniser by the current researchers. Physical characterization tests were carried out on 24 WiBE emulsions produced using an ultrasonic bath, with water percentage of $9 \%, 12 \%$ and $15 \%$, HLB value of $6,7,8$ and 9 , and surfactant dosage of $5 \%$ and $10 \%$. The water droplets produced using ultrasonic homogeniser were found to be evenly distributed and generally smaller in size. The density and viscosity values of these emulsions were found to be uniformly larger than WiBE produced using mechanical homogeniser. Emulsions with $15 \%$ water exceeded the density threshold, indicating the limits of the amount of water which can be added to the biodiesel fuel. Also, it was found that for viscosity, the HLB is limited to HLB 9 for higher surfactant dosage.
\end{abstract}

\section{Introduction}

Fuel modification technique is preferred to reduce emission level because the target emissions levels can be achieved using existing diesel engine with significant lower costs compared to engine based improvements [1]. An example of fuel modification techniques is the utilization of fuel emulsions such as water-in-diesel emulsion. Studies by Suresh et al. [2] had proved the effectiveness of using water-in-diesel emulsion in reducing various exhaust emissions. The study by Khalid 2017 et al. [3] claims that the oxygen content in biodiesel is about $11 \%$ to $15 \%$. The presence of oxygen in biodiesel assists in attaining lesser CO and HC compared to diesel, improves combustion efficiency but boosts the formation of NOx. This, however can be addressed by adding water into the content. The presence of water

*Corresponding author: ambri@utp.edu.my 
lowers the adiabatic flame temperature as it acts as heat sink [4]. Water-in-diesel emulsions have gained attention due to their potential in lowering NOx and particulate emissions [3].

In generating water-in-diesel emulsion, emulsifiers or surfactants are used to combine two immiscible solutions to produce the emulsions. These compounds reduce the surface tensions between the two fluids, activate their surfaces and maximize their superficial contact areas to make oil-in-water or water-in-oil two-phase emulsions [5]. The surfactants available on the market are categorized based on Hydrophilic-Lipophilic Balance (HLB) values. HLB values lower than 9 tends to be suitable for forming water-in-diesel emulsion [6].

Mechanical homogenization is the most common technique in the industry to produce water-in-diesel emulsions. This technique utilizes direct physical force to destroy the interfacial tension between the continuous and dispersed phases of the fluids to break big liquid droplet into uniform distribution and smaller sizes. Ultrasonic homogenization also utilized mechanical energy but in the form of ultrasonic sound waves. The technique is already widely applied in food and medical industries [6]. The lower the frequency of the ultrasonic waves, the higher the destructiveness to emulsion [Kojima 2014]. According to Lin and Chen [7], the emulsion produced using ultrasonic vibrator has smaller dispersed water droplet which is evenly distributed in continuous phase. These emulsions also have lower separation rate of dispersed water droplet from emulsion, higher emulsion stability and viscosity compare to the emulsions produced using mechanical homogenizer. Their research also found that the emulsions produced from ultrasonic technique showed other favourable characteristics including lower fuel consumption rate and $\mathrm{CO}$ emission due to a more intensive micro-explosion phenomenon, while having larger black smoke opacity [7].

The stability of the emulsion is mainly depending on the type and amount of surfactant used during the formulation of the emulsion. During the formation of emulsion, the mixture starts to change in the form of sedimentation, creaming and other time-dependent process [5]. Viscosity is another important property for water-in-diesel emulsion which affects the fuel combustion performance. The lesser the emulsion viscosity, the better the fuel fluidity, which indirectly improves fuel atomization thus improve fuel consumption and reduce exhaust emission [7]. Besides, the droplet size of the emulsion plays an important role in influencing the strength of micro-explosion process which critically affects the efficiency of the burning process [1].

According to the Protocol for Preparation of Nanoparticle Dispersions using ultrasonic disruption [9], indirect sonication is deliver when using an ultrasonic bath in which ultrasonic waves travel through the bath liquid and pass through the wall of the sample container before reaching the emulsion. The total amount of energy (E) delivered to the mixture depends on the applied power $(\mathrm{P})$ and the amount of time $(\mathrm{t})$ of the sonication process [9] stated in equation (1).

$$
E=P \times t
$$

The frequency of the ultrasonic waves affects the cavitation volume of the emulsion during the process. According to the research article by Hielscher [10], the lower the frequency of the ultrasonic waves, the higher the destructiveness to solid particles.

\section{Methodologies}

The methodologies include preparation of the various water-in-biodiesel emulsions, capturing images of the emulsions for water droplet size determination, measurements of density, viscosity and elementary analysis. 


\subsection{WiBE preparation}

A total of $24 \mathrm{WiBE}$ emulsions of $100 \mathrm{ml}$ were produced for characterization works using Honda SANPA W-118 ultrasonic bath. B5 biodiesel (a blend of 5\% palm methyl ester into diesel) was used to produce $\mathrm{WiBE}$ in this research. Table 1 shows the WiBE blends of the study. These emulsions were selected with reference to previous research of the current researchers to provide fair comparison. The defining parameters of the WiBE emulsions variations are water percentage of $9 \%, 12 \%$ and $15 \%$, HLB value of $6,7,8$ and 9 with surfactant dosage of 5\% and 10\%. Surfactants which consist of Tween-85 and Span-80 were initially mixed to produce the required surfactant HLB prior to introducing into the mixture during the homogenizing process. These emulsions were produced under the same conditions in the ultrasonic bath with frequency set at $45 \mathrm{kHz}$, for 30 minutes, power at $450 \mathrm{~W}$ and temperature at $43-49^{\circ} \mathrm{C}$.

Table 1. WiBE parameters.

\begin{tabular}{|c|c|c|c|}
\hline Sample ID & Water \% & HLB Value & Surfactant Dosage (\%) \\
\hline $\begin{array}{c}24 \text { emulsions } \\
\text { (WiBE 1 - WiBE 24) }\end{array}$ & $9,12,15$ & $6,7,8,9$ & 5,10 \\
\hline
\end{tabular}

\subsection{WiBE characterization}

The characterization of the emulsions includes the determination of water droplet size and distribution by examining the images taken using Olympus BX61 Optical Microscope, density was measured at $15^{\circ} \mathrm{C}$ using DA-645 Density Meter, while viscosity was determined at $40^{\circ} \mathrm{C}$ using DHR-1 Hybrid Rheometer.

The water droplet sizes and distribution were calculated using Sauter Mean Diameter, $d_{32}$ (SMD) following equation (2),

$$
d_{32}=\frac{\sum n_{i} D_{i}^{3}}{\sum n_{i} D_{i}^{2}}
$$

where $D_{i}$ is the diameter of the water droplet, and $n_{i}$ is the number of droplets with similar diameter value.

\section{Results and discussion}

The results obtained from the current work using ultrasonic homogeniser were compare to the previous findings of the same researchers which uses mechanical homogeniser.

\subsection{Water droplet size and distribution}

Figure 1 shows a comparison of images between the emulsions WiBE-17 (12-7-10), produced using (a) ultrasonic homogeniser and (b) mechanical homogeniser. WiBE prepared by ultrasonic homogenizer has shown to have a larger number of smaller dispersed-phase droplets that were more evenly distributed in the oil phase and a lower separation rate of dispersed water droplet making it more stable, compared to the emulsion than WiDE prepared by mechanical homogenizer. 


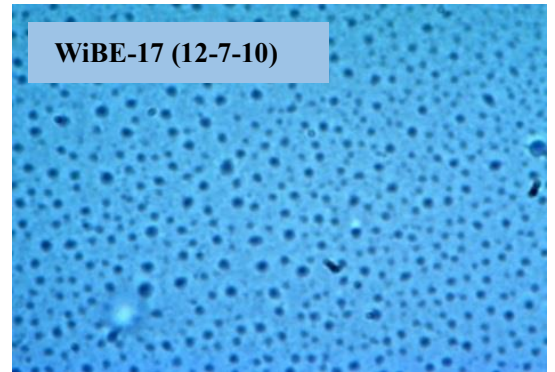

(a) Ultrasonic Homogeniser

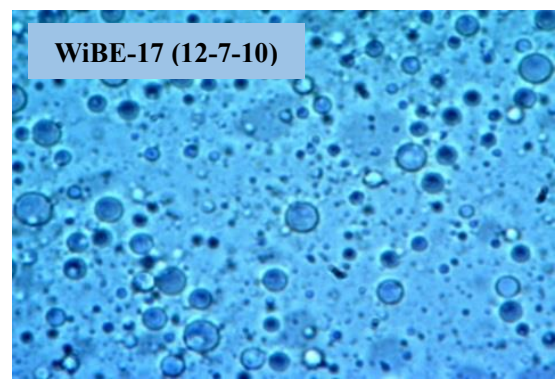

(b) Mechanical Homogeniser

Fig. 1. Images of water droplets and distribution

Measurement records of the emulsions SMD are shown Figure 2. All of the ultrasonic homogenised emulsions have SMD values of less than $2 \mu \mathrm{m}$, hovering around $1.14 \mu \mathrm{m}$ to $1.96 \mu \mathrm{m}$, as compared to emulsions produced using mechanical homogenizer, which have value ranging from $1.96 \mu \mathrm{m}$ to $6.91 \mu \mathrm{m}$. 18 out of 24 emulsions produced using ultrasonic homogenizer showed significant reduction in SMD value. Although some of the emulsions recorded increasing SMD, the increments were relatively insignificant to the decrement in values in majority of the emulsions.

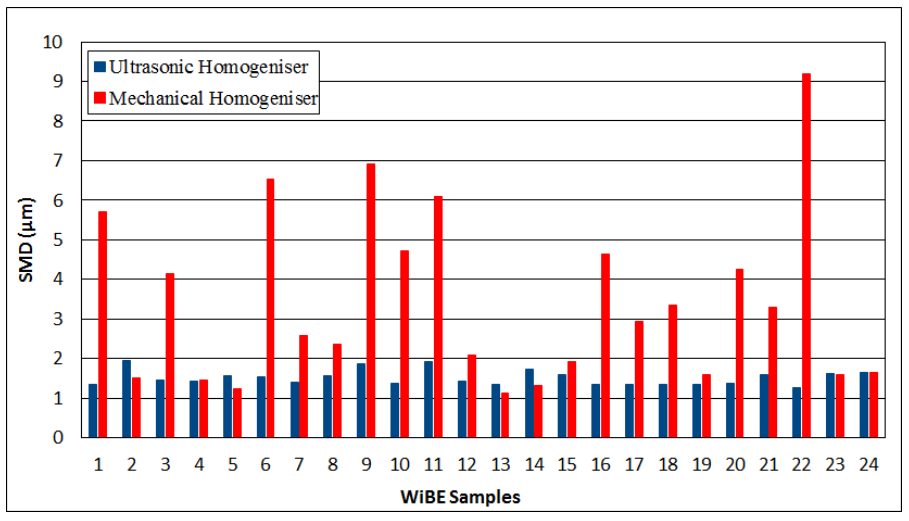

Fig. 2. SMD of WiBE emulsions using ultrasonic homogeniser and mechanical homogeniser.

\subsection{Effects on SMD}

The effects of adding water, variations in HLB values and surfactant dosages on SMD are depicted in Figure 3. In general, increasing percentage of water from $9 \%$ to $15 \%$ increases the SMD. For $9 \%$ and $15 \%$ water, the SMD are relatively constant when HLB increases from HLB 6 to HLB 9, while for 12\% water showed higher SMD at HLB 6 and HLB 9. For the effects of increasing surfactant dosage from 5 to $10 \%$, most emulsions showed a decreasing tends in SMD, except for 15\% water at HLB 6 and HLB 9. From the observation, HLB 7 produced more favourable SMD characteristics with insignificant influence of water and surfactant dosage. 


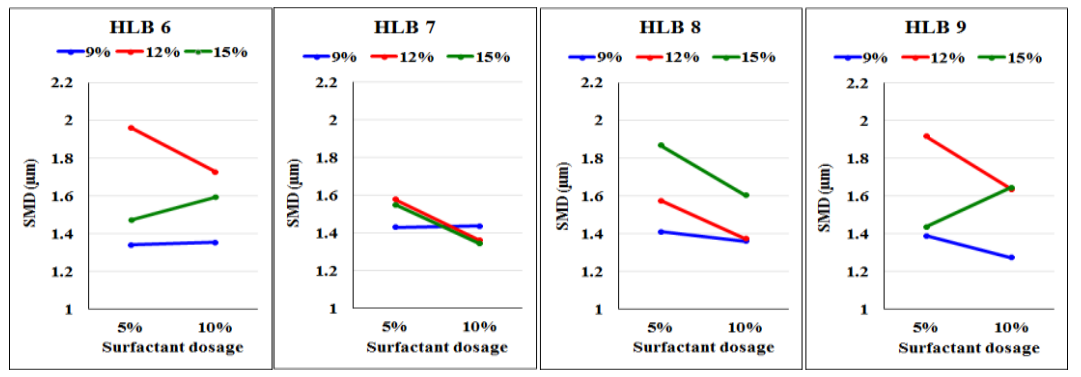

Fig. 3. Charts of SMD values vs surfactant dosage at various HLB and percentage of water

\subsection{Effects on density}

Figure 4 shows the effects of adding water, change in HLB values and surfactant dosage on the emulsion density. Ultrasonic homogenized emulsions are observed to produced emulsion with higher density. This is due to the smaller water particles and significantly more water droplets being incorporated into the emulsion during the homogenizing process. Water percentage of $15 \%$ in general exhibited high density, causing WiBE 6, 9 and 24 to exceed the maximum allowable emulsion density, while WiBE 15, 18 and 21 are in the upper level.

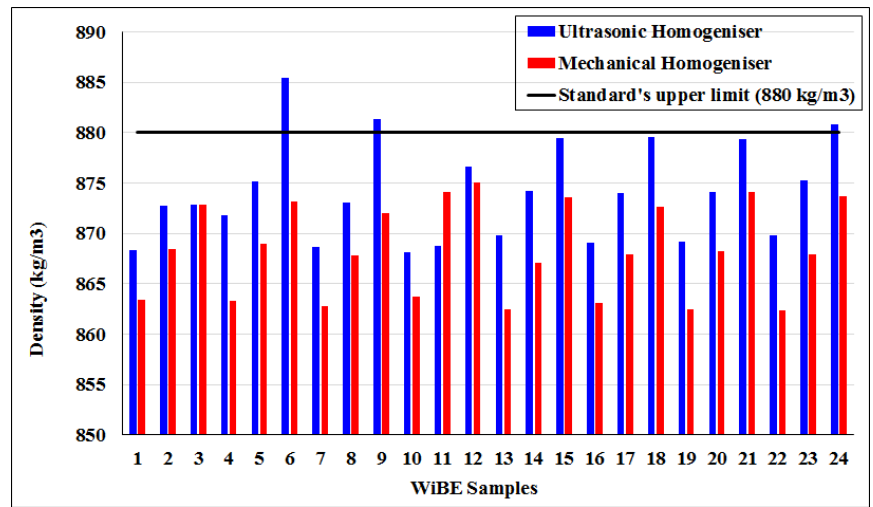

Fig. 4. Density vs WiBE emulsions using ultrasonic homogeniser and mechanical homogeniser.

Figure 5 shows the effects of increasing water percentage, HLB and surfactant dosage on the emulsions density. Similar to Figure 4, increasing water into the emulsions increases the density. Increasing surfactant dosage also has the effect of increasing the emulsion density, while HLB value has no significant effect on density. All emulsions of $9 \%$ and $12 \%$ water were within the allowable density values.

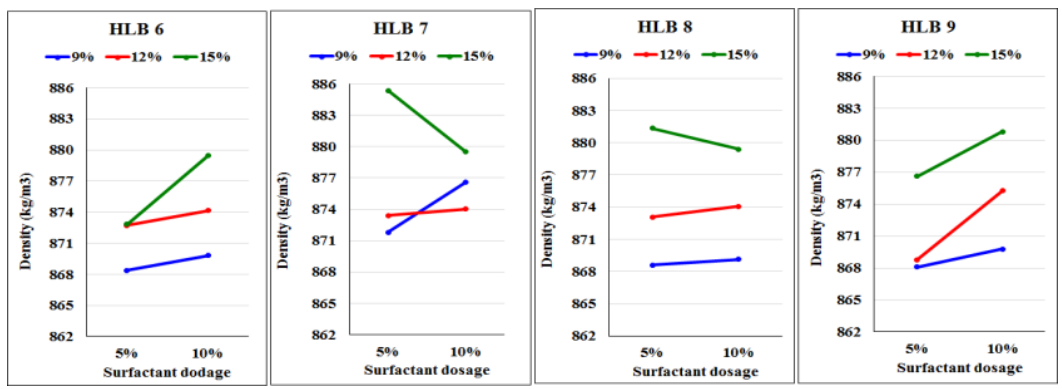

Fig. 5. Chart of density vs surfactant dosage at various HLB and percentage of water 


\subsection{Effects on viscosity}

Figure 6 shows that the minimum and maximum viscosity values of emulsions produced using ultrasonic homogenizer were $4.853 \mathrm{~mm}^{2} / \mathrm{s}$ and $10.452 \mathrm{~mm}^{2} / \mathrm{s}$ respectively. Comparatively, the minimum and maximum viscosity values of emulsions produced using mechanical homogenizer were $3.948 \mathrm{~mm}^{2} / \mathrm{s}$ and $5.394 \mathrm{~mm}^{2} / \mathrm{s}$ respectively. All 24 emulsions showed considerably higher viscosity value than their equivalent emulsions from previous research. According to Italy and France Water/Diesel Emulsions standards, the specifications for viscosity is from $2.5 \mathrm{~mm}^{2} / \mathrm{s}$ to $7.0 \mathrm{~mm}^{2} / \mathrm{s}$. The value of WiBE 22, 23 and 24 were above the acceptable range and significantly higher than other emulsions. The viscosity of WiBE 12 fell unusually above the trend therefore the results was considered faulty.

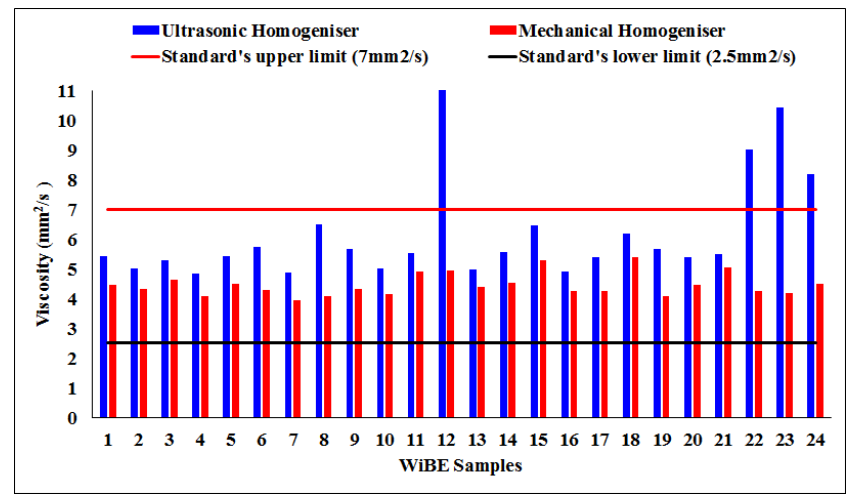

Fig. 6. Viscosity vs WiBE emulsions using ultrasonic homogeniser and mechanical homogeniser.

Figure 7 shows that increases in HLB value and surfactant dosage did little effects to the viscosity value, except for HLB 9 and 10\% surfactant dosage. WiBE emulsions of HLB 6, 7 and 8 were within the standard values. Nevertheless, viscosity value generally increases as water percentage increases.

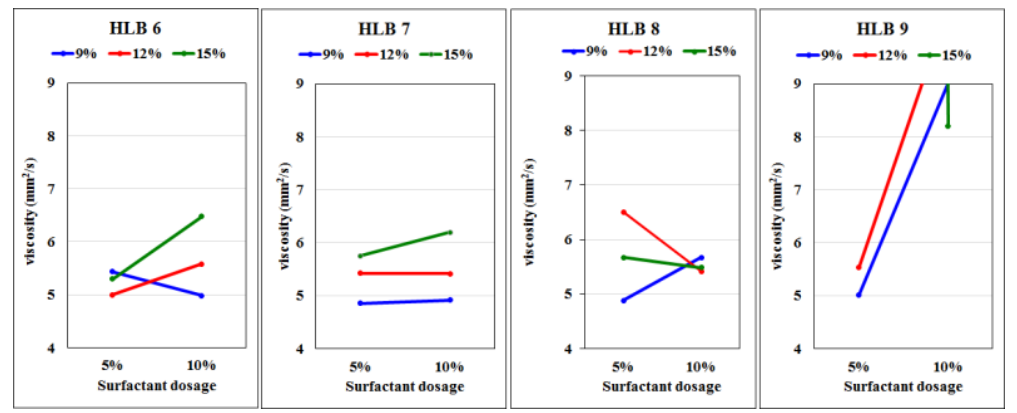

Fig. 7. Charts of HLB, Viscosity vs Surfactant dosage

\section{Conclusions}

24 water-biodiesel emaulsions with water percentage of $9 \%, 12 \%$ and $15 \%$, HLB value of 6 , 7,8 and 9 , and surfactant dosage of $5 \%$ and $10 \%$ were produced using an ultrasonic bath. The SMD values obtained for emulsions produced using ultrasonic homogenizer were generally smaller than emulsions produced using mechanical homogenizer. All of the emulsions showed SMD less than $2 \mu \mathrm{m}$. The water droplets were found to be evenly distributed in its continuous phase in most emulsions. The density and viscosity were found to be uniformly higher than WiBE emulsions produced using mechanical homogenizer. Emulsions with $15 \%$ water 
exceeded the density threshold, indicating the limits of the amount of water which can be added to the biodiesel fuel. Also, it was found that for viscosity, the HLB is limited to HLB 9 for higher surfactant dosage.

Further investigations to reduce viscosity while maintaining small water droplets of WiBE is suggested. These measures include the reduction in sonication time and the changing the ultrasonic frequency during the WiBE production, and optimising the amount of surfactant concentrations.

The authors thank Universiti Teknologi PETRONAS for the facility support and Ministry of Higher Education, Malaysia for the financial support through the Fundamental Research Grant Scheme (FRGS 1/2017/TK07/UTP/02/04).

\section{References}

1. A. M. Ithnin, H. Noge, H. A. Kadir and W. Jazair, Journal of the Energy Institute 87, 273-288 (2014).

2. V. Suresh, S. Vijayakumar, B. Varun and K. S. Amirthagadeswaran, Int. J. Appl. Environ. Sci. 9, 2739-2749 (2014).

3. Khalid A., Suardi M., Ronny Y. S. C., and Amirnordin S. H., Energy Procedia 111, 877884 (2017).

4. Mohammed Yahaya Khan, Z. A. Abdul Karim, H. Ftwi Yohaness, A. R. Abdul Aziz and I. M. Tan, The Scientific World Journal 2014, 527472 (2014).

5. D. Scarpete, Machines, Technologies, Materials, no. 7/2013 (2013).

6. H. Patil and J. Waghmare, International Research Journal of Engineering and Technology 4, 7, 1200-1204 (2017).

7. C.-Y. Lin and L.-W. Chen, Fuel 87, 2154-2161 (2008).

8. Y. Kojima, H. Imazu and K. Nishida, Ultrasonics Sonochemistry 21, 2, 722-728 (2014).

9. J. Taurozzi, V. Hackley and M. Wiesner, National Institue of Standards and Technology Materials Science and Engineering 2012).

10. T. Hielscher, European Nano Systems 2005, France, (2005). 